\title{
Diseño y Construcción de un Canal Hidráulico de Pendiente Variable
}

\author{
Hermes E. Castellanos ${ }^{(1)}$, Carlos A. Collazos ${ }^{(1)}$, Javier C. Farfan ${ }^{(2)}$, Farid Meléndez-Pertuz ${ }^{(3)}$ \\ (1) Univ. Manuela Beltrán, Grupo de Ciencias Básicas y Laboratorios, Bogotá D.C. - Colombia \\ (e-mail: hsajca@gmail.com; cacollazos@gmail.com) \\ (2) Universidad Nacional, Lab. de Nuevos Materiales, Bogotá D.C. -Colombia \\ (e-mail: jacf09@yahoo.com.mx) \\ (3) Universidad de La Costa, Grupo de Investigación en Electrónica, Barranquilla- Colombia \\ (e-mail: fmelende1@cuc.edu.co)
}

Recibido Mar. 27, 2017; Aceptado May. 24, 2017; Versión final Jul. 26, 2017, Publicado Dic. 2017

\begin{abstract}
Resumen
En este artículo se presenta el diseño, construcción, calibración y validación a través de prácticas experimentales usando un canal hidráulico de pendiente variable. El equipo forma parte del Laboratorio de Hidráulica de la Universidad Manuela Beltrán sede Cajicá en Colombia. El equipo se compone de un soporte estructural en aluminio de 5 metros de largo, un banco hidráulico de 250 litros, un canal rectangular en acrílico de 5 metros de largo, sensores de caudal y presión de bajo costo. Como novedad en comparación a otros prototipos similares, se incluyó un sistema hidráulico acoplado a un sensor de presión para registrar los cambios de presión en diferentes zonas del canal con mayor grado de precisión. Esta herramienta brindará apoyo a estudiantes, docentes e investigadores en los campos de hidráulica, ingeniería ambiental y áreas afines. Finalmente, la validación del funcionamiento del canal se da mediante la reproducción exitosa de experimentos propuestos en la literatura.
\end{abstract}

Palabras clave: canales abiertos; pendiente variable; borde libre; caudal; presión; flujo; energía específica; flujo laminar

\section{Design and Construction of a Hydraulic Channel of Variable Slope}

\begin{abstract}
In this article the design, construction, calibration and validation through experimental assays using a hydraulic channel of variable slope, is presented. This device is part of the Hydraulics Laboratory of the University Manuela Beltrán, campus Cajicá in Colombia. The experimental setup consists of a structural aluminum support of $5 \mathrm{~m}$ long, a hydraulic bench of 250 liters, a rectangular acrylic channel of 5 meters long, and low-cost flow and pressure sensors. As a novelty, and different from other similar laboratories, a hydraulic system which was coupled to a pressure sensor for recording pressure changes in different areas of the channel with a higher degree of accuracy was included. This tool will provide the necessary support to students, teachers and researchers in the fields of hydraulics, environmental engineering and related areas. Finally, performance validation of the channel was provided by the successful reproduction of experiments proposed in the literature.
\end{abstract}

Keywords: open channels; variable slope; free edge; caudal; pressure; flow; specific energy; laminar flow 


\section{INTRODUCCIÓN}

Los fluidos son elementos líquidos o gaseosos que al tener baja cohesión molecular adoptan la forma del recipiente que los contiene. Estos recipientes pueden clasificarse en canales o tuberías. En los primeros se tiene una superficie del fluido en contacto con la atmósfera, en el segundo el fluido está confinado en un espacio cerrado haciendo que este ejerza una presión a la tubería. En el caso de los fluidos líquidos, la diferencia para los conductos no solo se limita a la forma, sino al comportamiento del fluido dentro del conducto. Dentro de las tuberías el líquido fluye debido a fuerzas como son la gravedad, resistencia, presión y viscosidad; adicional a estas fuerzas, en los canales actúan la tensión superficial y fuerzas que puedan provocarse si hay sedimentos arrastrados. La adición de fuerzas en los canales con respecto a las tuberías hace que su estudio sea más complejo (Sotelo-Ávila, 2002).

Los canales adicionalmente tienen ciertas características que deben considerarse para entender la dinámica del fluido que contienen. Una de estas características es el borde libre, que se define como la distancia o altura desde la superficie del líquido (normalmente agua) hasta la parte superior del canal. El borde libre se tiene en cuenta ya que permite (entre otras condiciones), que las ondas de agua oscilen sin salirse del canal. Otra característica es la pendiente longitudinal del fondo del canal necesaria para el flujo del agua (Chow, 2004). Estas y otras características deben ser asumidas a la hora de la construcción de canales para la conducción de agua.

Los canales pueden ser de tipo natural o artificial debido a su origen. Los primeros han sido creados por los ecosistemas para drenar el agua de la tierra en forma de arroyos o ríos. Por su parte los canales artificiales son creados por el hombre para generar electricidad, navegación, riego, etc., estos canales tienen como principal reto conducir eficientemente el agua para cumplir la función por la cual fueron creados. Los canales naturales tienen su flujo en lo que se denomina "cauce"; este es irregular por las variaciones del caudal de agua en el tiempo, lo cual hace que las dimensiones, profundidad y forma varíen a lo largo del canal. Contrario a los canales naturales, los artificiales tienden a ser de forma geométrica claramente definidas y en diversas secciones se tienen dimensiones constantes.

Basados en las características anteriormente mencionadas, se observa que transportar agua en canales es un trabajo complejo que involucra muchas variables. Estos temas merecen especial consideración en tierras bajo riego. Este problema adquiere un nivel importante de complejidad cuando se trata del diseño de canales sin revestir excavados en suelos aluviales. Ello se debe a la gran cantidad de variables a tener en cuenta y a la falta de un conocimiento adecuado en lo que respecta a los fenómenos físicos vinculados (Farias, 1995).

En la literatura es posible encontrar el desarrollo de diferentes sistemas de canales o el estudio de los fenómenos físicos que intervienen en el flujo del agua por los canales, todo esto para la optimización de los recursos. Sistemas de adquisición de datos inalámbricos y acceso remoto para el cubrimiento de necesidades básicas como el desarrollado por (Bolaños et al, 2015), el cual permite aprovechar al máximo las fuentes naturales de agua y de esta manera evitar pérdidas considerables. Otro trabajo es la implementación de un sistema de automatización basado en controladores no lineales de nivel, para el control de las compuertas de un canal de irrigación (Dulhoste et al, 2007; Marbello, 2005).

La optimización de canales basados en su diseño es un tema de interés; (Vatankhah, 2013) optimiza el flujo a través de secciones geométricas semi-rectangulares que son propuestas y evaluadas para dicho fin por Swamee y Chahar (2012) y Tofiq y Guven (2015) quienes describen formas para el diseño óptimo de canales de riego desde la fuente al destino, minimizando costos teniendo en cuenta la topografía del canal y el uso de técnicas de programación lineal. Se han propuesto algoritmos como el MHBMO (Niazkar y Afzali, 2015) el cual pretende minimizar los costos y aumentar la optimización de secciones de canales de formas comunes. Por último, el análisis de flujos en canales sirve para apoyar actividades académicas, como es el caso de (Bougamouza et al., 2015), donde se realiza el análisis de la consecuencia de tener en el flujo un obstáculo en el fondo del canal rectangular, esto realizado en pruebas en el laboratorio. También se han diseñado y construido canales hidráulicos para aulas educativas como es el caso de (Marín, Menjívar y Zavaleta, 2012), trabajo en el que se generan manuales de mantenimiento y guías de laboratorio para el aprovechamiento del canal construido. En este trabajo se realiza un prototipo para el estudio académico, el cual cuenta con un sistema llamado "flauta", que fue acoplado a un sensor de presión de alta resolución que permite obtener la presión de la columna de agua antes y después de un cambio de área, causado por la ubicación de bloques con diferentes formas geométricas en cualquiera de las zonas seleccionadas a lo largo del canal. Por otro lado, el equipo en general le brinda al experimentador la familiaridad con los sistemas de adquisición de datos, a través del control de las variables de entrada y salida al sistema. 


\section{CANALES HIDRÁULICOS.}

El flujo de agua en un conducto puede ser: flujo en canal abierto o flujo en tubería. Aunque estas dos clases de conductos son similares en muchos aspectos, se diferencian por el hecho que los canales tienen una superficie libre en la cual el líquido está en contacto con la atmósfera. Por otra parte, el flujo en tuberías puede ser: a tubo lleno funcionando bajo presión (o forzado); y a tubo parcialmente lleno funcionando como un canal (Marín, Menjívar y Zavaleta, 2012).
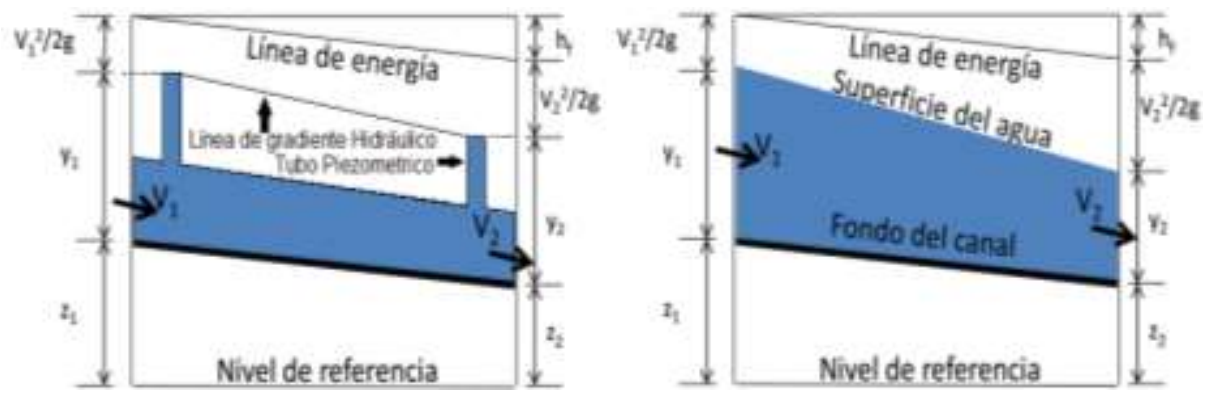

Fig. 1: Comparación entre tuberías a flujo lleno y flujo en canales abiertos (Adaptada de Marín, Menjívar y Zavaleta, 2012)

A pesar de la similitud que existe entre estos dos tipos de flujo, es mucho más difícil resolver problemas de flujo en canales abiertos que en tuberías a presión. Las condiciones de flujo en canales abiertos se complican por el hecho de que la posición de la superficie libre puede cambiar con el tiempo y el espacio, y también porque la profundidad de flujo, caudal y las pendientes del fondo del canal y de la superficie libre son interdependientes (Chow, 2004). En la figura 1 se muestra el comparativo entre ambos flujos.

\section{Clases de Canales Abiertos}

Un canal abierto es un conducto en el cual el agua fluye con una superficie libre, dependiendo de su origen, un canal puede ser natural o artificial. Los canales naturales incluyen todos los cursos de agua que existen de manera natural en la tierra, los cuales varían en tamaño desde pequeños arroyos en zonas montañosas, hasta quebradas, ríos grandes y pequeños ciénagas y los mares. Los canales artificiales son aquellos construidos o desarrollados por los seres humanos un ejemplo de ellos son: canales de vegetación, canales de centrales hidroeléctricas, canales y canaletas de irrigación, cunetas de drenaje, vertederos, canales de desborde, canales de madera, así como canales de modelos construidos en el laboratorio con propósitos experimentales (Chow, 2004; Marín, 2012). También existe un caso particular de ciudades que sufren de inundaciones repentinas por lluvias, donde sus calles se convierten en canales (Cama-Pinto et al., 2016).

\section{DISEÑO Y CONSTRUCCIÓN DE CANALES ABIERTOS}

En este ítem se describe el proceso de cálculo de parámetros del canal partiendo de ecuaciones existentes para poder realizar su diseño. Entre los parámetros calculados están la geometría del canal, la curva de pendiente crítica y la profundidad normal.

\section{Geometría del Canal}

Apoyándose en las necesidades presentes del laboratorio de hidráulica de la Universidad Manuela Beltrán y con base a las características del banco hidráulico (Armfield), se optó por la construcción de un canal rectangular que cumpla con las especificaciones sugeridas en la figura 2. Estos detalles se toman como referentes del canal de manufactura original que es complemento del banco ya existente en la institución.

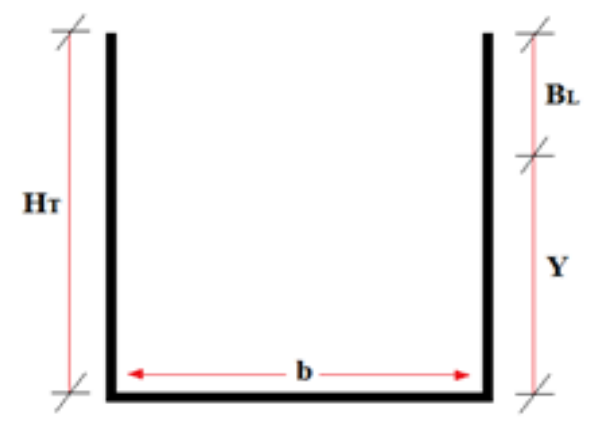

Fig. 2: Geometría del canal. 
De la literatura se toman las ecuaciones a utilizar para la obtención de los valores propios del canal (Chow, 2004; Rodríguez, 2008; Morales Nava y Et al, 2013). En la figura 2, se observan las dimensiones propuestas para el diseño, tales como: ancho del canal $(b)=0,076$ metros; altura del canal $(\mathrm{HT})=0,25$ metros; y aunque no se observa en la figura 2 , también se tiene la longitud del canal $(L)=5$ metros.

El borde libre (BL): se obtiene por una simple regla de 3 teniendo en cuenta que este sería el $30 \%$ de la altura total del canal.

$$
\mathrm{B}_{\mathrm{L}}=\frac{(30 \%) \times(0,25 \mathrm{~m})}{100 \%} \Rightarrow \mathrm{B}_{\mathrm{L}}=0,075 \mathrm{~m}
$$

La profundidad hidráulica o de flujo (Y) es:

$$
\mathrm{H}_{\mathrm{T}}=\mathrm{B}_{\mathrm{L}}+\mathrm{Y} \Rightarrow \mathrm{Y}=\mathrm{H}_{\mathrm{T}}-\mathrm{B}_{\mathrm{L}}=0,25 \mathrm{~m}-0,075 \mathrm{~m} \Rightarrow \mathrm{Y}=0,175 \mathrm{~m}
$$

Cálculos Geométricos:

El área $(A)$ del canal es:

$$
A=b \times Y=(0,076 m) \times(0,175 m) \Rightarrow A=0,0133 \mathrm{~m}^{2}
$$

Perímetro mojado $(\mathrm{P})$ :

$$
\mathrm{P}=\mathrm{b}+2 \mathrm{Y}=(0,076 \mathrm{~m})+(2 \times 0,175 \mathrm{~m}) \Rightarrow \mathrm{P}=0,426 \mathrm{~m}
$$

Radio hidráulico $(\mathrm{R})$ :

$$
\mathrm{R}=\frac{\mathrm{A}}{\mathrm{P}}=\frac{\mathrm{b} \times \mathrm{Y}}{\mathrm{b}+(2 \times \mathrm{Y})} \Rightarrow \mathrm{R}=\frac{0,0133 \mathrm{~m}^{2}}{0,426 \mathrm{~m}} \Rightarrow \mathrm{R}=0,031 \mathrm{~m}
$$

Factor de sección (Z):

$$
\mathrm{Z}=\mathrm{b} \times \mathrm{Y}^{1,5}=(0,076 \mathrm{~m}) \times(0,175 \mathrm{~m})^{1,5} \Rightarrow \mathrm{Z}=5,56 \times 10^{-3} \mathrm{~m}
$$

De la ecuación de Manning tenemos:

$$
\mathrm{V}=\frac{\mathrm{R}^{\frac{2}{3}} \times \mathrm{S}^{\frac{1}{2}}}{\eta}
$$

Dónde: $\mathrm{R}=$ radio hidráulico $(\mathrm{m}) ; \mathrm{S}=$ pendiente del canal (adimensional); y $\eta=$ coeficiente de rugosidad (adimensional).

El caudal $\left(\mathrm{m}^{3} / \mathrm{s}\right)$ se define como:

$$
\mathrm{Q}=\mathrm{V} \times \mathrm{A}
$$

Donde: $\mathrm{V}=$ es la velocidad del agua al interior del canal $(\mathrm{m} / \mathrm{s}) ; \mathrm{A}=$ área transversal del canal $\left(\mathrm{m}^{2}\right)$.

Al reemplazar la ecuación (7) en (8) obtenemos otra expresión para el caudal, la cual se utilizará más adelante:

$$
\Rightarrow \mathrm{Q}=\frac{\mathrm{A} \times \mathrm{R}^{2 / 3} \times \mathrm{S}^{1 / 2}}{\eta}
$$

Ahora, el factor de sección también se da por:

$$
\mathrm{Z}=\frac{\mathrm{Q}}{\sqrt{\mathrm{g}}}
$$

Finalmente, al igualar las ecuaciones (6) y (10), reemplazando el valor de (b) y el valor de la fuerza gravitacional al nivel del mar, $g=9,8 \mathrm{~m} / \mathrm{s}^{2}$ respectivamente; se llega a la ecuación (11) que será utilizada junto a la ecuación (9), para determinar la pendiente crítica del canal (Sc) adimensional.

$$
\Rightarrow \mathrm{Q}=0,238 \mathrm{Y}^{1,5}
$$




\section{Gráfica de la Curva de Pendiente contra el Caudal Crítico}

Para determinar la curva de la pendiente crítica versus caudal, el rango de altura crítica $Y_{c}(m)$ y posteriormente el caudal de operación del canal $Q_{o p}$, es necesario realizar iteraciones con las ecuaciones (9) y (11), y luego obtener el valor del punto crítico directamente del grafico $S_{c}$ vs $Q_{c}$.

Las iteraciones realizadas se muestran en la Tabla 1 . En ella se dan valores de profundidad del flujo $\mathrm{Y}_{\mathrm{c}}$, en un rango de 0,005 a 0,25 metros y se utiliza $\eta=0,010$ que es la rugosidad del vidrio; estos datos serán utilizados para obtener la gráfica que permita obtener el punto de operación del equipo (Chow, 2004).

Tabla 1: Iteraciones para obtener el punto de operación del canal.

\begin{tabular}{|c|l|l|l|l|l|}
\hline Profundidad " $Y_{c}{ }^{\prime}(\mathrm{m})$ & Caudal " $\mathrm{Q}_{c}{ }^{\prime}\left(\mathrm{m}^{3} / \mathrm{s}\right)$ & Factor & $\begin{array}{c}\text { Pendiente Critica "Sc" } \\
\text { (adimensional) }\end{array}$ & $S c \times 10^{-3}$ & $\begin{array}{c}\text { Caudal }\left(Q_{c}\right) \\
\times 10^{-3}\end{array}$ \\
\hline 0,006 & 0,00011061 & 0,00242828 & 0,00207496 & 2,07496185 & 0,1106124 \\
\hline$\ldots$ & $\ldots$ & $\ldots$ & $\ldots$ & $\ldots$ & $\ldots$ \\
\hline 0,01 & 0,000238 & 0,00548502 & 0,00188277 & 1,88277209 & 0,238 \\
\hline 0,02 & 0,00067317 & 0,01601977 & 0,00176576 & 1,76575849 & 0,67316566 \\
\hline 0,03 & 0,00123668 & 0,02923739 & 0,00178912 & 1,78912406 & 1,23668428 \\
\hline 0,04 & 0,001904 & 0,04417664 & 0,00185758 & 1,85758403 & 1,904 \\
\hline 0,2 & 0,02128737 & 0,34842154 & 0,00373279 & 3,73279295 & 21,2873671 \\
\hline 0,21 & 0,02290371 & 0,36871937 & 0,00385851 & 3,85851235 & 22,9037133 \\
\hline$\ldots$ & $\ldots$ & $\ldots$ & $\ldots$ & $\ldots$ & $\ldots$ \\
\hline 0,22 & 0,02455902 & 0,38906917 & 0,00398445 & 3,98444944 & 24,5590169 \\
\hline 0,23 & 0,02625238 & 0,40946549 & 0,00411058 & 4,11057721 & 26,2523818 \\
\hline 0,24 & 0,02798297 & 0,42990362 & 0,00423687 & 4,23687293 & 27,9829708 \\
\hline 0,25 & 0,02975 & 0,45037945 & 0,00436332 & 4,36331737 & 29,75 \\
\hline
\end{tabular}

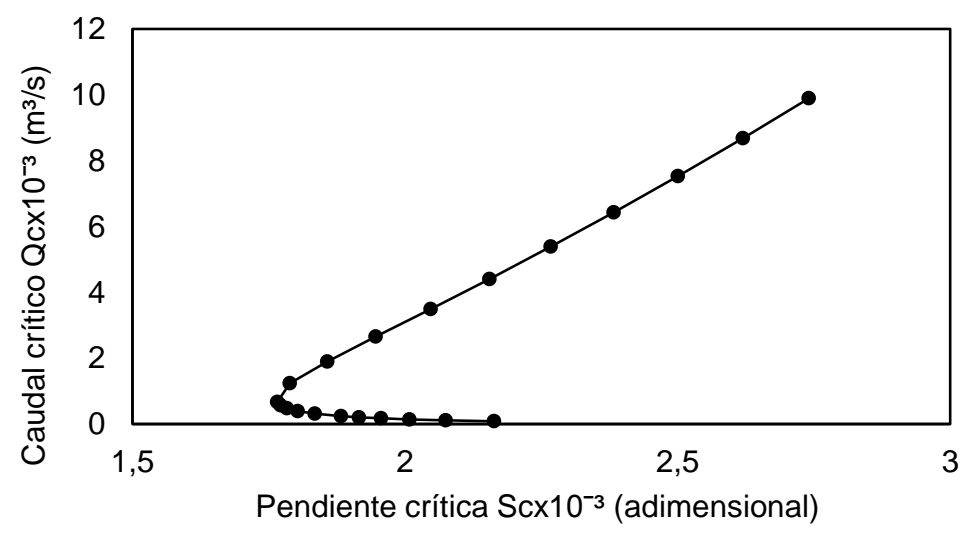

Fig. 3: Curva para encontrar el caudal máximo de operación del canal.

Al realizar las iteraciones y graficar, se evidencia que existe una parte de la gráfica terminada en punta con forma de nariz, y es precisamente en el cambio de pendientes sobre la curva donde podemos ubicar el caudal de operación máxima de nuestro sistema, como se puede ver en la figura 3 , de donde se obtienen los valores siguientes: profundidad crítica $\left(\mathrm{Y}_{\mathrm{C}}=0,02 \mathrm{~m}\right)$; pendiente crítica $\left(\mathrm{S}_{\mathrm{c}}=0,001765\right)$; y caudal crítico $\left(\mathrm{Q}_{\mathrm{c}}=\right.$ $\left.0,00067317 \mathrm{~m}^{3} / \mathrm{s}\right)$.

Por lo tanto, el caudal de operación estará dado por:

$$
\mathrm{Q}=\frac{\mathrm{Q}_{\mathrm{C}} \times \mathrm{Y}_{\max }}{\mathrm{Y}_{\mathrm{c}}}=\frac{\left(0,00067317 \mathrm{~m}^{3} / \mathrm{s}\right) \times 0,175 \mathrm{~m}}{0,02 \mathrm{~m}} \Rightarrow \mathrm{Q}_{\mathrm{op}}=5,890 \times 10^{-3} \mathrm{~m}^{3} / \mathrm{s}
$$

Por último, determinamos la velocidad de operación de nuestro canal:

$$
\mathrm{Q}_{\mathrm{op}}=\mathrm{V}_{\mathrm{op} *} \mathrm{~A} \therefore \mathrm{V}_{\mathrm{op}}=\frac{\mathrm{Q}_{\mathrm{op}}}{\mathrm{A}}=\frac{5,890 \times 10^{-3} \mathrm{~m}^{3} / \mathrm{s}}{0,0133 \mathrm{~m}^{2}} \Rightarrow \mathrm{V}_{\mathrm{op}}=0,4428 \mathrm{~m} / \mathrm{s}
$$

Corrección de Profundidad - Caudal de operación (Qop) - Velocidad "Crítica": 


$$
\begin{aligned}
& \mathrm{Y}_{\mathrm{C}_{\mathrm{c}}}=\sqrt[3]{\frac{\mathrm{Qop}^{2}}{\mathrm{~g} \times \mathrm{b}^{2}}}=\sqrt[3]{\frac{\left(5,890 \times 10^{-3} \mathrm{~m}^{3} / \mathrm{s}\right)^{2}}{\left(9,81 \mathrm{~m} / \mathrm{s}^{2}\right) \times(0,076 \mathrm{~m})^{2}}} \Rightarrow \mathrm{Y}_{\mathrm{C}_{\mathrm{c}}}=0,0849 \mathrm{~m} \\
& \mathrm{~V}_{\mathrm{C}_{\mathrm{c}}}=\sqrt[3]{\frac{\mathrm{Qop} \times \mathrm{g}}{\mathrm{b}}}=\sqrt[3]{\frac{\left(5,890 \times 10^{-3} \mathrm{~m}^{3} / \mathrm{s}\right)^{2} \times\left(9,81 \mathrm{~m} / \mathrm{s}^{2}\right)}{0,076 \mathrm{~m}}} \Rightarrow \mathrm{V}_{\mathrm{C}_{\mathrm{c}}}=0,9127 \mathrm{~m} / \mathrm{s} \\
& \mathrm{Q}_{\mathrm{C}_{\mathrm{c}}}=\mathrm{A}_{\mathrm{C}_{\mathrm{c}}} \times \mathrm{V}_{\mathrm{C}_{\mathrm{c}}}=(0,076 \mathrm{~m}) \times(0,0849 \mathrm{~m}) \times(0,9127 \mathrm{~m} / \mathrm{s}) \Rightarrow \mathrm{Q}_{\mathrm{C}_{\mathrm{c}}}=5,889 \times 10^{-3} \mathrm{~m}^{3} / \mathrm{s}
\end{aligned}
$$

\section{Cálculo de Profundidad Normal}

Para el cálculo de esta profundidad se utiliza nuevamente el método de iteración haciendo uso de las ecuaciones mostradas en (Streeter y et al, 2000) las cuales se detallan en a continuación:

$$
\begin{aligned}
& \mathrm{A}=\mathrm{C} \times \mathrm{P}^{\frac{2}{5}}=\mathrm{b} \times \mathrm{Y}_{\mathrm{n}} \\
& \mathrm{P}=\mathrm{b}+2 \mathrm{Y}_{\mathrm{n}} \\
& \mathrm{C}=\left[\frac{\mathrm{Qop} \times \mathrm{n}}{\mathrm{S}^{1 / 2}}\right]^{3 / 5}=\left[\frac{\left(5,890 \times 10^{-3}\right) \times(0,01)}{0,01765^{1 / 2}}\right]^{3 / 5} \Rightarrow \mathrm{C}=0,00975
\end{aligned}
$$

(S), representa la pendiente del canal y el valor usado en nuestro caso es 0,01765 (adimensional).

Reemplazando la ecuación (18) y (19) en (17) tenemos finalmente la ecuación (20) que permitirá, por medio de iteraciones, encontrar el valor de la profundidad normal.

$$
0,00975 \times\left(0,076 \mathrm{~m}+2 \mathrm{Y}_{\mathrm{n}}\right)^{\frac{2}{5}}=0,076 \mathrm{~m} \times \mathrm{Y}_{\mathrm{n}}
$$

Después de realizar el proceso de iteración se obtiene que: $Y_{n}=0,0693236 \mathrm{~m}$

Ahora, retomando los valores encontrados, se tiene que: $\mathrm{Y}_{\mathrm{C}_{\mathrm{c}}}=0,0849 \mathrm{~m} ; \mathrm{Y}_{\mathrm{n}}=0,0693236 \mathrm{~m} ; \mathrm{Y}_{\max }=0,175 \mathrm{~m}$

De los resultados mostrados anteriormente se observa que $\mathrm{Y}_{\mathrm{n}}<\mathrm{Y}_{\mathrm{C}_{\mathrm{c}}}$, lo que indica que el canal es de pendiente fuerte "zona 2", pero de igual manera cabe resaltar que el perfil del flujo puede cambiar con el valor del caudal utilizado, esto indica que en el mismo canal se pueden obtener diversos tipos de flujo: sub-critico, crítico y supercrítico.

La inclinación del canal se calcula con ayuda del teorema de Pitágoras, teniendo en cuenta que el ángulo será de $\theta=1^{\circ}$ y $\mathrm{L}=5 \mathrm{~m}$ como se muestra en la Figura 4.

$$
\mathrm{h}=5 \mathrm{~m} \times \tan 1^{\circ}=0,0872 \mathrm{~m}
$$

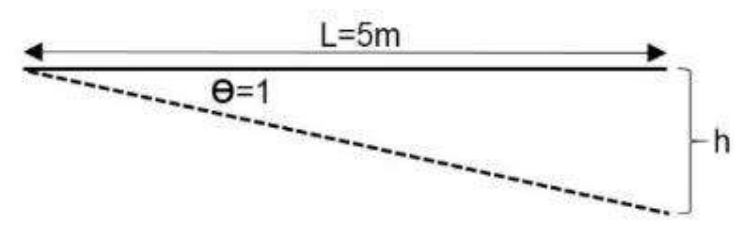

Fig. 4: Inclinación (h) del canal

\section{PLANOS DEL DISEÑO, SENSORES Y PROTOTIPO FINAL}

En la figura 5 se observa el diseño del prototipo. En la figura 6 se muestran las cotas del prototipo. La figura 7 muestra los sensores utilizados. En la figura 8 se muestra una fotografía del prototipo final. La Tabla 2 detalla las dimensiones generales del canal. 


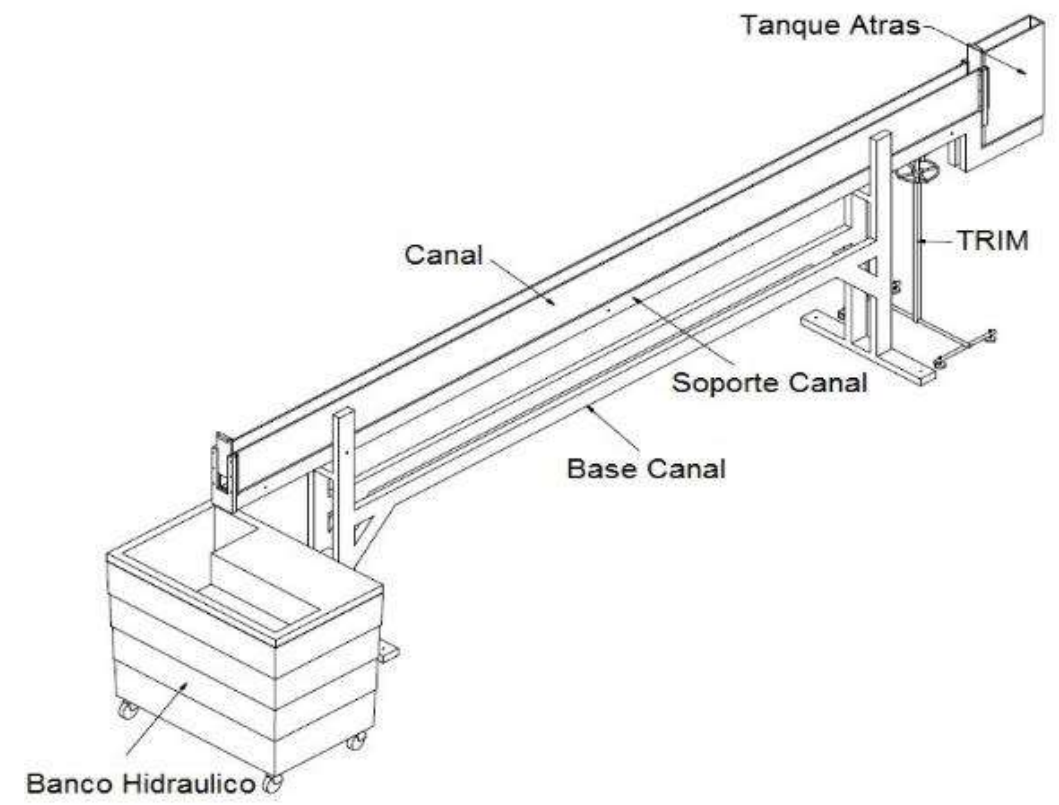

Fig. 5: Diseño del prototipo

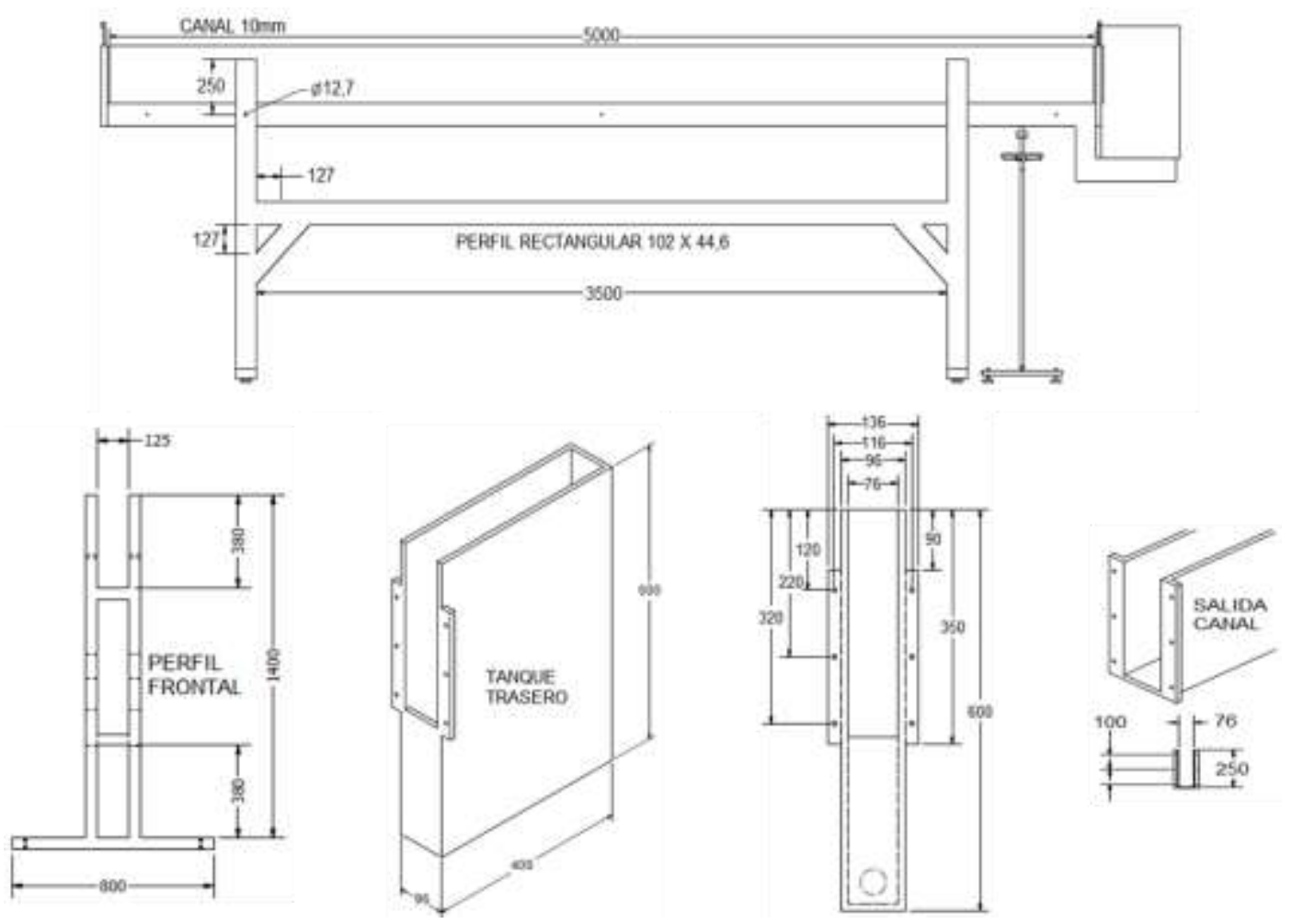

Fig. 6: Cotas del prototipo
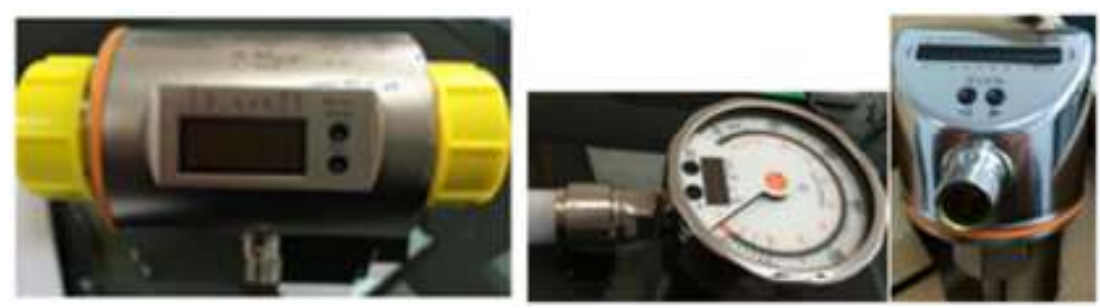

Fig. 7: Sensor de caudal, presión y flujo respectivamente 


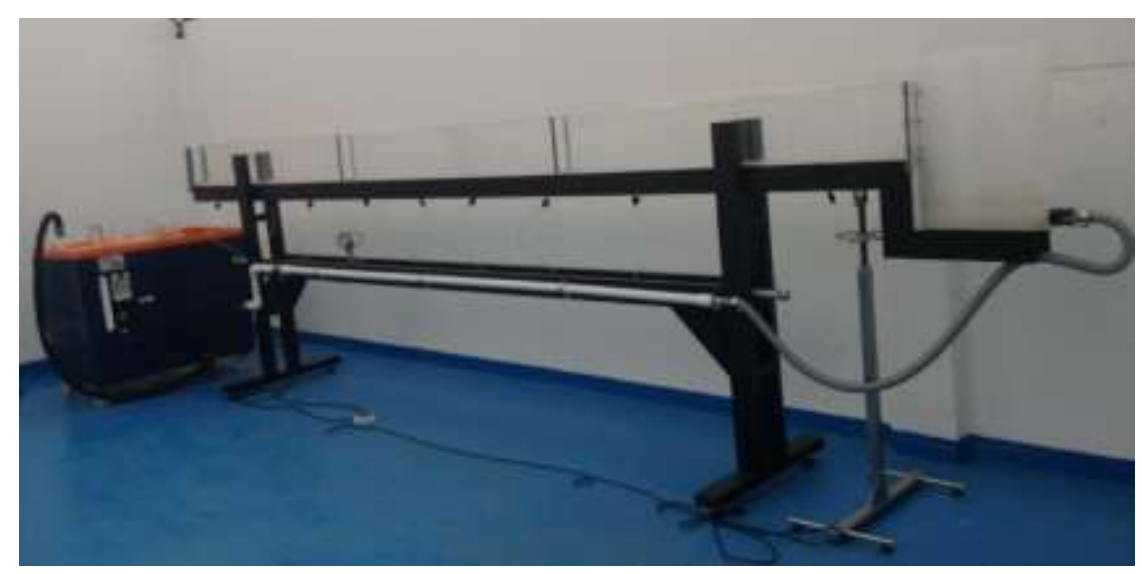

Fig. 8: Prototipo

Tabla 2: Dimensiones generales del Canal

\begin{tabular}{|l|c|}
\hline Longitud & $L=5 \mathrm{~m}$ \\
\hline Ancho & $b=0,076 \mathrm{~m}$ \\
\hline Altura de la canal & $H=0,25 \mathrm{~m}$ \\
\hline Angulo máximo de inclinación & $S_{\max }=0,017=1^{\circ}$ \\
\hline Rango de operación del canal & $Q=0-5,890 \times 10^{-3} \mathrm{~m}^{3} / \mathrm{s}$ \\
\hline Variación de velocidad del canal & $v=0-0,4428 \mathrm{~m} / \mathrm{s}$ \\
\hline Variación del nivel del agua & $h=0-0,175 \mathrm{~m}$ \\
\hline Variación de pendiente del canal & $S=0-0,1765=0-1^{\circ}$ \\
\hline
\end{tabular}

\section{RESULTADOS Y DISCUSIÓN}

Para efectos de validar el funcionamiento del prototipo construido, se realizó un experimento que permitió determinar la relación entre la energía específica y la carga de agua por encima y por debajo de un bloque con forma de triángulo sumergido en el canal, para ello se utilizó el montaje mostrado en la figura 9. Para efectos de encontrar la energía especifica antes y después del bloque no se contemplan los cambios bruscos en el flujo debido a que no hay cambio en la geometría de la sección que dé lugar a tirantes críticos múltiples (Sotelo-Ávila et al., 2006).

De (Mejía, 2008; Marbello, 2005), es importante recordar que la energía específica se define como la suma de la energía potencial (la cual está relacionada directamente con la profundidad del flujo) y la energía cinética (que no es más que la velocidad de la columna de agua); esto matemáticamente es:

$$
\mathrm{E}=\mathrm{y}+\frac{\mathrm{V}^{2}}{2 \mathrm{~g}}
$$

En función del caudal es:

$$
\mathrm{E}=\mathrm{y}+\frac{\mathrm{Q}^{2}}{2 \mathrm{gy}^{2}}
$$

Dónde: $E=$ energía específica $(\mathrm{m}) ; y=$ profundidad de flujo $(\mathrm{m}) ; Q=$ caudal del flujo $\left(\mathrm{m}^{3} / \mathrm{s}\right)$ y $g=$ fuerza gravitacional al nivel del $\operatorname{mar}=9,81 \mathrm{~m} / \mathrm{s}^{2}$.

Con el montaje de la figura 8, se utilizaron 3 caudales $Q_{1}=0,00013333 \mathrm{~m}^{3} / \mathrm{s}, Q_{2}=0,00046111 \mathrm{~m}^{3} / \mathrm{s}, Q_{3}=$ $0,00058333 \mathrm{~m}^{3} / \mathrm{s}$ para cada uno de los experimentos realizados, estas medidas fueron obtenidas directamente del sensor de caudal. Para cada valor de $Q$ se tomaron valores de altura antes y después del bloque con el fin de observar el comportamiento del flujo de agua, estos valores fueron reportados con el vernier, el cual ofrece una precisión de $+/-0,05 \mathrm{~mm}$. 


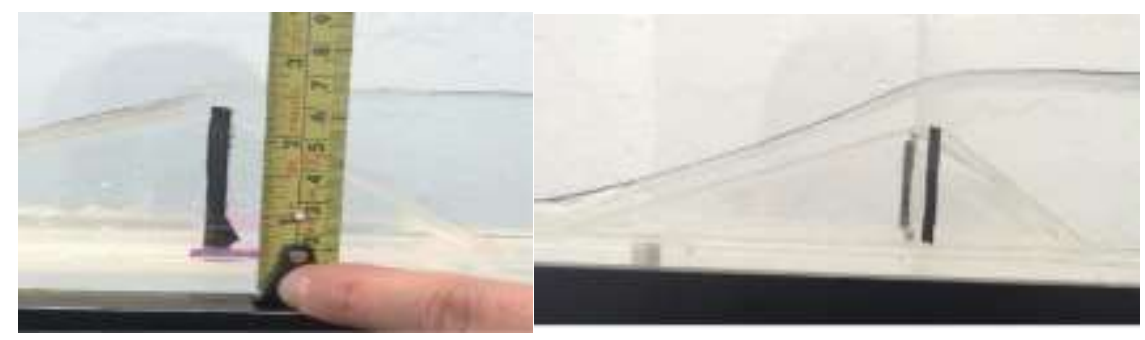

Fig. 9: Comportamiento del flujo de agua en el canal

A continuación, se presentan las tablas de datos y las familias de curvas obtenidas para los valores de $Q$, respectivamente en la Figura 10.

Tabla 3: Datos Energía y profundidad para $Q_{1}$

\begin{tabular}{|l|l|}
\hline $\mathrm{E}(\mathrm{m})$ & $\mathrm{y}(\mathrm{m})$ \\
\hline 0,060549807 & 0,0605 \\
\hline 0,054661153 & 0,0546 \\
\hline 0,045687674 & 0,0456 \\
\hline 0,030695975 & 0,0305 \\
\hline 0,011760072 & 0,0099 \\
\hline$\ldots$ & $\ldots$ \\
\hline 0,032821628 & 0,00245 \\
\hline 0,046086156 & 0,046 \\
\hline 0,058553271 & 0,0585 \\
\hline 0,099018601 & 0,099 \\
\hline 0,0950202 & 0,095 \\
\hline
\end{tabular}

Tabla 4: Datos Energía y profundidad para $Q_{2}$

\begin{tabular}{|l|l|}
\hline \multicolumn{1}{|c|}{$E(\mathrm{~m})$} & $y(\mathrm{~m})$ \\
\hline 0,071037446 & 0,0706 \\
\hline 0,069359299 & 0,0689 \\
\hline 0,054261774 & 0,0535 \\
\hline 0,043141955 & 0,0419 \\
\hline 0,03292858 & 0,0306 \\
\hline$\ldots$ & $\ldots$ \\
\hline 0,029019742 & 0,011 \\
\hline 0,038080649 & 0,0086 \\
\hline 0,052696866 & 0,0069 \\
\hline 0,053953736 & 0,0068 \\
\hline 0,04363808 & 0,0078 \\
\hline
\end{tabular}

Tabla 5: Datos Energía y profundidad para $Q_{3}$

\begin{tabular}{|l|l|}
\hline \multicolumn{1}{|c|}{$\mathrm{E}(\mathrm{m})$} & \multicolumn{1}{c|}{$\mathrm{y}(\mathrm{m})$} \\
\hline 0,073065701 & 0,0724 \\
\hline 0,05903729 & 0,058 \\
\hline 0,05045333 & 0,049 \\
\hline 0,039033623 & 0,0364 \\
\hline 0,030209406 & 0,0243 \\
\hline$\ldots$ & $\ldots$ \\
\hline 0,043191365 & 0,0103 \\
\hline 0,050426902 & 0,0092 \\
\hline 0,04266188 & 0,0104 \\
\hline 0,031803291 & 0,014 \\
\hline 0,031096647 & 0,0145 \\
\hline 0,04026996 & 0,0109 \\
\hline
\end{tabular}

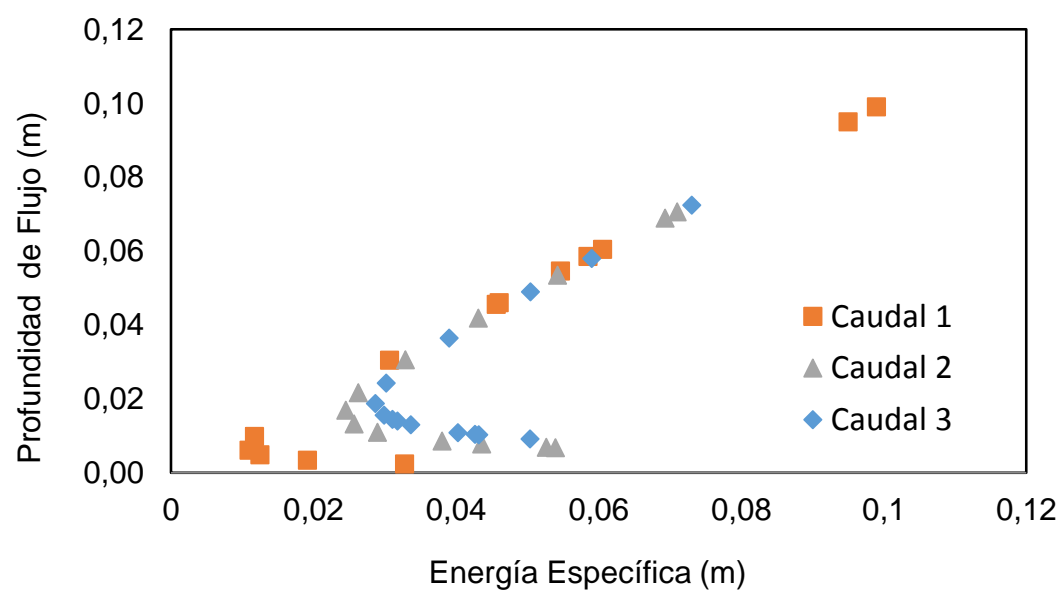

Fig. 10: Curva de energía específica a diferentes caudales " $Q$ " 
De la figura 10, se obtienen los valores experimentales de $Y_{c}$ y $E_{m}$ para cada uno de los experimentos realizados a caudal constante, estos valores según (Chow, 2004; Mejía, 2008;) se dan en el punto de inflexión de la curva de la gráfica y se detallan en la Tabla 6.

Tabla 6: Datos experimentales obtenidos de las curvas de energía específica

\begin{tabular}{|l|l|l|c|}
\hline Experimento 1 & $Q_{1}=0,00013333 \mathrm{~m}^{3} / \mathrm{s}$ & $Y_{C 1 E x p}=0,0061 \mathrm{~m}$ & $\mathrm{E}_{\mathrm{m} 1 \operatorname{Exp}}=0,0109937 \mathrm{~m}$ \\
\hline Experimento 2 & $\mathrm{Q}_{2}=0,00043111 \mathrm{~m}^{3} / \mathrm{s}$ & $\mathrm{Y}_{\mathrm{C} 2 \operatorname{Exp}}=0,0217 \mathrm{~m}$ & $\mathrm{E}_{\mathrm{m} 2 \operatorname{Exp}}=0,02633036 \mathrm{~m}$ \\
\hline Experimento 3 & $\mathrm{Q}_{3}=0,00058333 \mathrm{~m}^{3} / \mathrm{s}$ & $\mathrm{Y}_{\mathrm{C} 3 \operatorname{Exp}}=0,018 \mathrm{~m}$ & $\mathrm{E}_{\mathrm{m} 3 \operatorname{Exp}}=0,02867868 \mathrm{~m}$ \\
\hline
\end{tabular}

En la Tabla 6, se observa que existe una dependencia directa entre la energía específica y el caudal, es decir que, a mayor caudal, mayor energía especifica; de igual manera este fenómeno se evidencia en la figura 10, donde las curvas se van distanciando una de la otra en la medida en que " $Q$ " se incrementa tal como lo dice (Chow, 2004), esto permite evidenciar que el canal cumple con las expectativas del diseño. Esto será corroborado posteriormente con los cálculos realizados y que se muestran a continuación.

Para efectos de validar los resultados mostrados en Tabla, se aplican las ecuaciones para canales rectangulares, según (Chow, 2004; Rodríguez, 2008):

$$
\mathrm{E}_{\min }=\frac{2}{3} \mathrm{Y}_{\mathrm{c}}
$$

Con $\mathrm{q}=$ caudal por unidad de ancho, $\mathrm{Q}=$ caudal $\left(\mathrm{m}^{3} / \mathrm{s}\right)$ y $\mathrm{g}=9,81 \mathrm{~m} / \mathrm{s}^{2}$.

$$
\begin{aligned}
& Y_{c}=\sqrt[3]{\frac{q^{2}}{g}} \\
& q=\frac{Q}{b}
\end{aligned}
$$

De las ecuaciones (24), (25) y (26) se obtienen los valores teóricos que se registran en la tabla 7.

Tabla 7: Datos Teóricos para cada caudal "Q"

\begin{tabular}{|l|l|l|l|}
\hline Experimento 1 & $\mathrm{Q}_{1}=0,00013333 \mathrm{~m}^{3} / \mathrm{s}$ & $\mathrm{Y}_{\mathrm{C} 1 \mathrm{Teo}}=0,007143913 \mathrm{~m}$ & $\mathrm{E}_{\mathrm{m} 1 \mathrm{Teo}}=0,01071587 \mathrm{~m}$ \\
\hline Experimento 2 & $\mathrm{Q}_{2}=0,00046111 \mathrm{~m}^{3} / \mathrm{s}$ & $\mathrm{Y}_{\mathrm{C} 2 \mathrm{Teo}}=0,001633756 \mathrm{~m}$ & $\mathrm{E}_{\mathrm{m} 2 \mathrm{Teo}}=0,02450634 \mathrm{~m}$ \\
\hline Experimento 3 & $\mathrm{Q}_{3}=0,00058333 \mathrm{~m}^{3} / \mathrm{s}$ & $\mathrm{Y}_{\mathrm{C} 3 \mathrm{Teo}}=0,001910999 \mathrm{~m}$ & $\mathrm{E}_{\mathrm{m} 2 \mathrm{Teo}}=0,02866499 \mathrm{~m}$ \\
\hline
\end{tabular}

El error porcentual es calculado con:

$$
|\% \mathrm{E}|=100 *\left|\frac{\mathrm{V}_{\mathrm{Teo}}-\mathrm{V}_{\mathrm{Exp}}}{\mathrm{V}_{\mathrm{Teo}}}\right|
$$

En la Tabla 8, se reporta el porcentaje de error de $y_{c}$ y $e_{m}$ de cada experimento, teniendo en cuenta los datos consignados en las tablas 6 y 7 .

Tabla 8: Porcentaje de error obtenido en cada experimento para $\mathrm{Y}_{\mathrm{c}}$ y $\mathrm{E}_{\mathrm{m}}$.

\begin{tabular}{|l|c|c|}
\cline { 2 - 3 } \multicolumn{1}{c|}{} & \% error de $Y_{c}$ & \% error de $E_{m}$ \\
\hline Experimento 1 & 14,61 & 2,59 \\
\hline Experimento 2 & 32,82 & 7,44 \\
\hline Experimento 3 & 5,80 & 0,047 \\
\hline
\end{tabular}

Posteriormente, al retomar los datos de la tabla 5 , y realizar su respectiva gráfica, se aprecia el comportamiento de la energía específica antes y después del bloque, de igual forma se podrá observar como varía el flujo sobre, por encima y por debajo del punto de inflexión. 


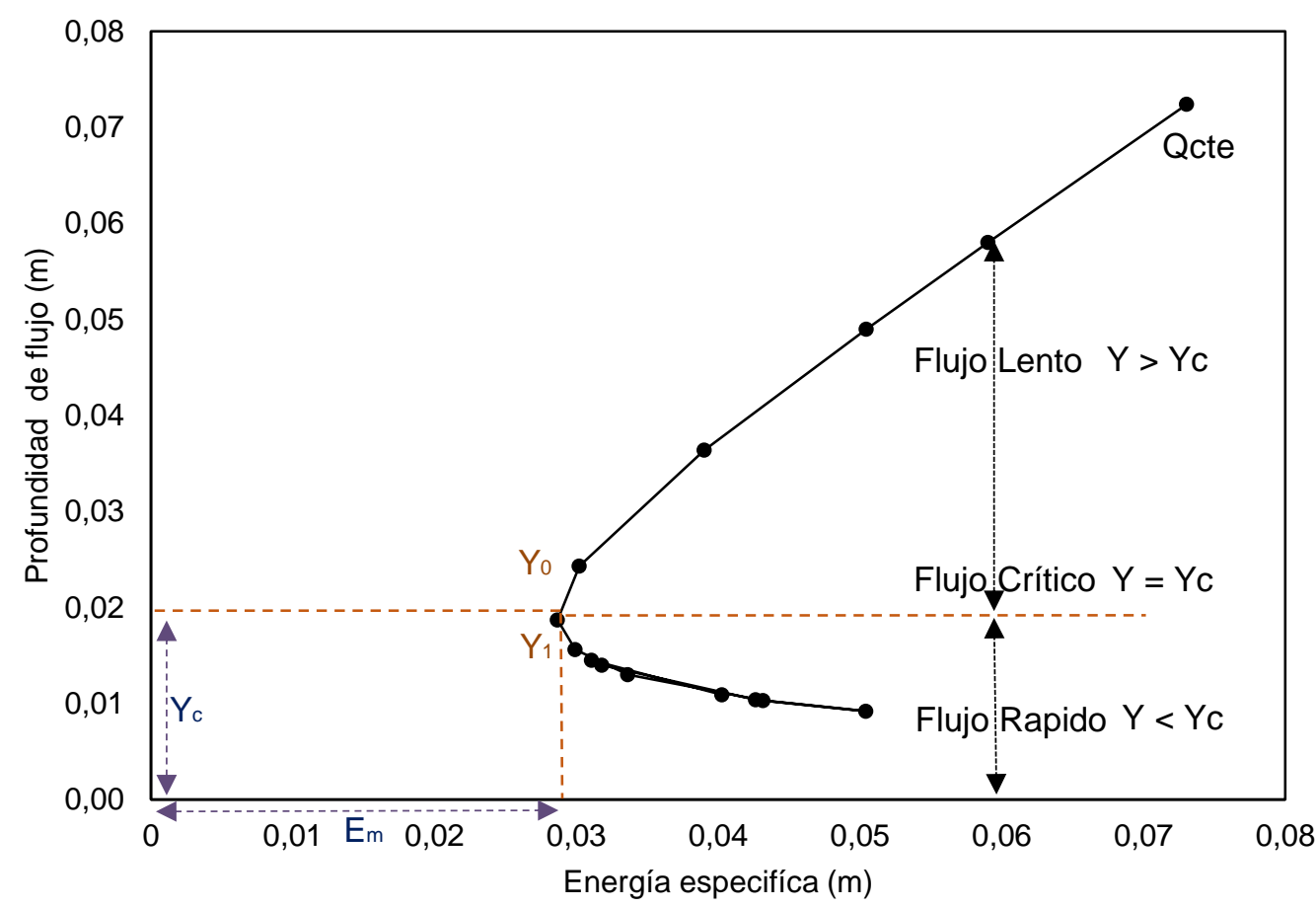

Fig. 11: Curva de energía específica a caudal Q constante

En la figura 11 se puede identificar que efectivamente la curva tiene simetría y esto se evidencia al revisar los puntos $Y_{0}$ y $Y_{1}$. Por otro lado, es importante tener en cuenta que se puede ganar o perder energía específica dependiendo de los rangos de profundidades utilizados, es decir si se está por encima o por debajo de la profundidad crítica $Y_{c}$, es por esta razón que se puede tener un flujo lento, crítico o rápido según (Mejía, 2008; Villón, 2007) en las zonas marcadas en la figura 11.

\section{CONCLUSIONES}

A partir de los resultados obtenidos, se puede indicar lo siguiente: 1) el diseño y construcción del canal de pendiente variable que incluye sensores para caudal y presión en tiempo real, representa una inversión de bajo costo en comparación con otros canales existentes; 2) la construcción del canal, también ha incrementado el libre desarrollo de prácticas de laboratorio de las áreas de hidráulica y fluidos de la Universidad Manuela Beltrán sede Cajicá, Bogotá - Colombia con una cobertura del 95\% del plan académico ofertado; 3) los resultados obtenidos como validación del prototipo, muestran que efectivamente el canal cumple con las condiciones necesarias para estudiar y validar diferentes fenómenos físicos que se puedan presentar a baja escala y logren ser utilizados como modelo para estudios reales; y 4) La implementación de los sistemas de instrumentación con que cuenta el canal, permite al estudiante familiarizarse con herramientas que algunos canales existentes no poseen y que dificultan la libre interpretación de los datos recolectados a lo largo de un experimento. Como trabajo a futuro se espera integrar todos los sensores con los que cuenta el canal a un PLC, con el fin de desarrollar laboratorios virtuales en tiempo real.

\section{REFERENCIAS}

Bolaños, M.D., T.S. López, Diseño de un sistema de adquisición y tratamiento de datos mediante comunicación inalámbrica para el canal rectangular hidráulico del laboratorio de ingeniería civil de Univalle, Journal Boliviano de Ciencias, ISSN: $2075-8936$ (en línea), 11(33), 27-39, 2015. https://goo.gl/hPBDxf. Acceso: 25 de febrero (2017)

Bougamouza, M., Bouhadef, M. y Zitoun, T., Contribution to Experiments of a Free Surface Supercritical Flow over an Uneven Bottom, International Scholarly and Scientific Research \& Innovation, 9(12), 2109-2113 (2015)

Cama-Pinto, A., Acosta-Coll, M., Piñeres-Espitia, G., Caicedo-Ortiz, J., Zamora-Musa, R. y Sepúlveda-Ojeda, J., Diseño de una red de sensores inalámbricos para la monitorización de inundaciones repentinas en la ciudad de Barranquilla, Colombia, doi: 10.4067/S0718-33052016000400005, Ingeniare. Revista chilena de ingeniería, 24(4), 581-599 (2016)

Chow, V.T., Hidráulica de Canales Abiertos, 1ª Ed., 667. McGraw Hill, Santafé de Bogotá, Colombia (1994) 
Dulhoste, J.F., Georges D., Besançon G. y Jerez C.J., Comparación de Controladores de Nivel para Canales Abiertos Basados en un Modelo por Colocación, doi: 10.4067/S0718-07642007000600003, Información Tecnológica (en línea), 18(6), 13-18 (2007)

Farias, H.D., Fórmulas prácticas para el diseño de canales sin revestir en terrenos aluviales, doi: 10.4995/ia.1995.2682, Ingeniería del Agua (en línea), 2(3), 53-69 (1995)

Marbello, P.R., Manual de Prácticas de Laboratorio de Hidráulica, (en línea: https://goo.gl/88qQtb, acceso: 25 de febrero de 2017), Universidad Nacional de Colombia, Sede Medellín, Colombia (2005)

Marín, C.A., Menjívar, M.J. y Zavaleta, J.M., Diseño y construcción de un canal hidráulico de pendiente variable para uso didáctico e investigación, Tesis de pregrado, Facultad de Ingeniería, Universidad de El Salvador (2012)

Morales, J.G. y Parra A., Mejoras al método usual de diseño hidráulico de alcantarillas. Ingeniería Hidráulica y Ambiental, ISSN: 1680-0338 (en línea), 34(1), 3-18, 2013. https://goo.gl/mifu5B. Acceso 25 de febrero (2017)

Mejía, F.J., Relación de las curvas de energía específica y pendiente de fricción con las zonas de flujo libre en canales, Revista EIA, ISSN: 1794-1237 (en línea), (9), 69-75, 2008. https://goo.gl/ZLEMCe. Acceso 25 de febrero (2017)

Niazkar, M. y Afzali, S.H., Optimum Design of Lined Channel Sections, doi: 10.1007/s11269-015-0919-9, Water Resources Management (en línea), 29(6), 1921-1932 (2015)

Swamee, P.K. y Chahar B.R., Optimal alignment of a canal route, doi: 10.1680/wama.11.00097, Water Management (en línea), 166(8), 422-431 (2012)

Sotelo-Ávila, G., Hidráulica de canales, 1ª Ed., 836. UNAM Facultad de Ingeniería, México DC, México (2002)

Sotelo G. y Cafaggi A., Criterios de energía específica mínima y momentum mínimo en el cálculo del régimen crítico en canales de sección compuesta, Ingeniería, investigación y tecnología, ISSN: 1405-7743 (en línea), 7(3), 175-184, 2006. https://goo.gl/pkYocr. Acceso 25 de febrero (2017)

Rodríguez, P., "Hidráulica de canales" 1ª Ed., 480. Ciudad de México, México (2008)

Streeter, V.L., Benjamin, E. y Keith W. "Mecánica de Fluidos", 9ª Ed., 508. McGraw Hill, Ciudad de México, México (2000)

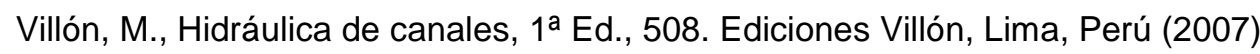

Tofiq, F.A. y Guven, A., Optimal design of trapezoidal lined channel with least cost: Semi-theoretical approach powered by genetic programming, doi: 10.4314/wsa.v41i4.07, Water SA (en línea), 41(4), 483-489 (2015)

Vatankhah, A.R., Semi-regular polygon as the best Hydraulic section in practice (Generalized solutions), doi: 10.1016/j.flowmeasinst.2014.05.016, Flow Measurement and Instrumentation (en línea), 38, 67-61 (2014) 